\title{
Oxytocin Modulation of Amygdala Functional Connectivity to Fearful Faces in Generalized Social Anxiety Disorder
}

\author{
Stephanie M Gorka ${ }^{1,2}$, Daniel A Fitzgerald ${ }^{1,3}$, Izelle Labuschagne ${ }^{4,5}$, Avinash Hosanagar, ${ }^{6,7}$, \\ Amanda G Wood ${ }^{8,9,10}$, Pradeep J Nathan ${ }^{4, I^{\prime}}$ and K Luan Phan*, , 2,3 \\ 'Department of Psychiatry, University of Illinois at Chicago, Chicago, IL, USA; '² Department of Psychology, University of Illinois at Chicago, Chicago, \\ IL, USA; ${ }^{3}$ Mental Health Service Line, Jesse Brown VA Medical Center, Chicago, IL, USA; ${ }^{5}$ School of Psychological Sciences, Monash University, \\ Melbourne, Victoria, Australia; ${ }^{5}$ School of Psychology, Australian Catholic University, Melbourne, Victoria, Australia; ${ }^{6}$ Department of Psychiatry, \\ University of Michigan, Ann Arbor, MI, USA; ${ }^{7}$ Mental Health Service, VA Ann Arbor Healthcare System, Ann Arbor, MI, USA; ${ }^{8}$ School of Psychology, \\ University of Birmingham, Birmingham, UK; ${ }^{9}$ Child Neuropsychology, Murdoch Children's Research Institute, Melbourne, Victoria, Australia; \\ ${ }^{10}$ Southern Clinical School, Monash University, Melboume, Victoria, Australia; ' 'Department of Psychiatry, University of Cambridge, Cambridge, UK
}

\begin{abstract}
The neuropeptide oxytocin (OXT) is thought to attenuate anxiety by dampening amygdala reactivity to threat in individuals with generalized social anxiety disorder (GSAD). Because the brain is organized into networks of interconnected areas, it is likely that OXT impacts functional coupling between the amygdala and other socio-emotional areas of the brain. Therefore, the aim of the current study was to examine the effects of OXT on amygdala functional connectivity during the processing of fearful faces in GSAD subjects and healthy controls (HCs). In a randomized, double-blind, placebo (PBO)-controlled, within-subjects design, 18 HCs and I7 GSAD subjects performed a functional magnetic resonance imaging task designed to probe amygdala response to fearful faces following acute intranasal administration of PBO or OXT. Functional connectivity between the amygdala and the rest of the brain was compared between OXT and $\mathrm{PBO}$ sessions using generalized psychophysiological interaction analyses. Results indicated that within individuals with GSAD, but not HCs, OXT enhanced functional connectivity between the amygdala and the bilateral insula and middle cingulate/dorsal anterior cingulate gyrus during the processing of fearful faces. These findings suggest that OXT may have broad pro-social implications such as enhancing the integration and modulation of social responses.

Neuropsychopharmacology (2015) 40, 278-286; doi: I0.1038/npp.2014.168; published online 6 August 20I4
\end{abstract}

\section{INTRODUCTION}

Generalized social anxiety disorder (GSAD), also known as generalized social phobia, is highly prevalent and characterized by extreme fear and avoidance of social situations (American Psychiatric Association, 1994). Research suggests that hyperactive threat response may be involved in the pathophysiology of GSAD (Schultz and Heimberg, 2008; Shin and Liberzon, 2010). For example, laboratory studies have shown that GSAD patients exhibit attentional biases for threatening social information (Foa et al, 2000; Mogg et al, 2004), and exaggerated affective responses to aversive social cues (Hermann et al, 2002; Roelofs et al, 2009). Heightened amygdala reactivity has been strongly linked to these social threat-related impairments and many have posited that the amygdala is an important therapeutic target for the management of GSAD (Labuschagne et al, 2010;

* Correspondence: Professor KL Phan, Department of Psychiatry, University of Illinois at Chicago, 1747 West Roosevelt Road, IJR/ WROB Suite 244, Chicago, IL 60608, USA, Tel: +3I2 355 5954, Fax: +312413 1703, E-mail: klphan@uic.edu

Received I6 April 2014; revised 30 June 2014; accepted I July 20 I4; accepted article preview online 7 July 2014
Phan et al, 2006). Indeed, amygdala hyperactivity to threat has been shown to be modulated by pharmacological treatments including selective serotonin reuptake inhibitors (Harmer et al, 2006) and benzodiazepines (Paulus et al, 2005). Importantly, however, current pharmacological treatments for GSAD are not universally effective and there is a critical need to identify novel, effective pharmacological interventions (Taylor et al, 2012).

Oxytocin (OXT), a neuropeptide produced in the hypothalamus, has been increasingly recognized as a potential therapeutic, anxiolytic agent. For years, OXT agonists have been shown to be effective in animal models of anxiety (Windle et al, 1997). More recently, studies in humans have demonstrated that OXT administration increases pro-social behaviors like trust, empathy, and willingness to take social risks (Kosfeld et al, 2005), whereas dampening psychophysiological responses to stressors (Ditzen et al, 2009). Although the mechanisms of these effects are unclear, it has been shown that OXT can inhibit neurons in the centromedial amygdala through its excitatory effects on $\gamma$-aminobutyric acid (GABA) inhibitory (GABAergic) projections that originate from the lateral and capsular part of the central nucleus of the amygdala, which disrupts integration of information and inhibits amygdala output to 
areas of the brain necessary for fear responding (Huber et al, 2005). Consistent with this evidence, functional magnetic imaging (fMRI) studies with healthy volunteers have shown that intranasal OXT administration dampens amygdala reactivity to threatening facial expressions (Domes et al, 2007; Kirsch et al, 2005), and naturalistic social interactions (Baumgartner et al, 2008).

We have extended these studies to examine the effects of OXT on amygdala reactivity to social threat in GSAD (Labuschagne et al, 2010) and found that OXT attenuated (or normalized) the hyperactive amygdala reactivity to fearful faces in GSAD patients. These results suggest that OXT has a specific effect on fear-related amygdala reactivity and may have an impact on GSAD patients by modulating neural responses to social signals of threat.

The amygdala is a good candidate target for OXT's effects given that, like other anxiolytic pharmacotherapies, OXT promotes GABAergic inhibition in the amygdala (Huber et al, 2005). However, brain regions are organized into networks of interconnected areas (Van Den Heuvel and Hulshoff Pol, 2010), and neuropeptides, such as OXT, lack spatial specificity and have receptors located throughout the brain. Researchers have therefore speculated that OXT affects not only specific regions but also the functional coupling between these regions (Bethlehem et al, 2013). Consequently, studies utilizing functional connectivity methods are greatly needed to elucidate the circuit-level effects of OXT.

To date, only a few studies have examined the effects of OXT on functional connectivity in humans (Dodhia et al, 2014; Domes et al, 2007; Riem et al, 2012; Sripada et al, 2013). Broadly, results suggest that OXT enhances connectivity between social-emotional areas of the brain, and reduces connectivity between regions implicated in fear responding. For example, during an interpersonal cooperation task, Rilling et al (2012) found that OXT increased functional connectivity between the amygdala and the anterior insula. Meanwhile, Kirsch et al (2005) found that OXT reduced functional coupling between the amygdala and the brainstem. Of note, prior studies have exclusively included healthy control (HC) subjects and no study to our knowledge has examined OXT-induced, task-dependent changes in functional connectivity in a patient group characterized by increased fear and anxiety such as GSAD. We have previously examined resting-state connectivity in patients with GSAD and demonstrated that OXT reversed or normalized the reduced amygdala-rostral anterior cingulate cortex functional connectivity observed under placebo (PBO; Dodhia et al, 2014). Given the differences in OXTinduced changes in connectivity between HCs and GSAD patients (Kirsch et al, 2005; Dodhia et al, 2014), it is especially critical to explore whether OXT modulates functional connectivity during actual social threat in this clinical population.

The current study is a novel analysis of existing fMRI data from our previous study on the neural effects of OXT in a sample of HC and GSAD subjects (Labuschagne et al, 2010). The study utilized a two-session (PBO vs OXT), doubleblind, within-subjects design. Our prior analyses, from the same cohort, indicated that GSAD patients exhibited hyperactive amygdala reactivity to fearful faces relative to HCs, which was normalized on OXT. These effects were not observed in response to angry or happy faces. Thus, in the present secondary analysis, we focused on examining OXT's effects on amygdala connectivity to fearful faces. We employed a generalized form of context-dependent psychophysiological interaction analyses (gPPI; McLaren et al, 2012), using the amygdala as the seed region of interest, to determine whether the effects of OXT on amygdala functional connectivity differed between groups (ie, HCs $v s$ GSAD). Given the literature reviewed above, we predicted that OXT's effects on amygdala functional connectivity to fearful faces would differ between HC and GSAD groups and that these differential effects would localize to areas relevant to socio-emotional functions.

\section{MATERIALS AND METHODS}

\section{Participants}

A total of 36 right-handed, male volunteers were enrolled in the study. Eighteen were considered HCs and eighteen met criteria for current GSAD. One control and two GSAD patients were excluded from the current study because of poor scan data quality. Group differences in demographics and clinical characteristics are presented in Table 1. GSAD diagnoses were confirmed using the Clinical International Diagnostic Interview-Version 2.1 (WHO, 1997), and probes taken from the Liebowitz Social Anxiety Scale (LSAS; Liebowitz, 1987). To ensure that GSAD patients were classified as the 'generalized' subtype, they were required to have a total LSAS score greater than 70 , and a 'social situations' subscale score greater than 30. GSAD participants were excluded if they had a co-occurring anxiety disorder, a depressive episode within the past six months, alcohol/substance use disorder within the past year, or any lifetime history of post-traumatic stress disorder, bipolar disorder, psychotic disorder, mental retardation, or developmental disorders. HCs had no lifetime

Table I Demographic and Clinical Characteristics

\begin{tabular}{lcc}
\hline & HC $(\boldsymbol{n}=\mathbf{I 7})$ & GSAD $(\boldsymbol{n}=\mathbf{I 6})$ \\
\hline Age (years) & $29.9(10.5)^{\mathrm{a}}$ & $29.8(9.1)^{\mathrm{a}}$ \\
Education & $16.0(2.5)^{\mathrm{a}}$ & $14.7(1.6)^{\mathrm{a}}$ \\
BDI-II & $1.3(1.9)^{\mathrm{a}}$ & $11.2(7.5)^{\mathrm{b}}$ \\
BAI & $2.2(5.0)^{\mathrm{a}}$ & $17.1(8.4)^{\mathrm{b}}$ \\
State anxiety (STAI) & $23.6(4.4)^{\mathrm{a}}$ & $38.9(16.3)^{\mathrm{b}}$ \\
Trait anxiety (STAI) & $27.4(5.3)^{\mathrm{a}}$ & $51.1(11.5)^{\mathrm{b}}$ \\
LSAS: total & $13.9(8.3)^{\mathrm{a}}$ & $83.3(16.6)^{\mathrm{b}}$ \\
LSAS: performance & $6.89(5.1)^{\mathrm{a}}$ & $39.5(9.7)^{\mathrm{b}}$ \\
LSAS: social situations & $7.06(4.6)^{\mathrm{a}}$ & $43.56(8.6)^{\mathrm{b}}$ \\
\hline
\end{tabular}

Abbreviations: BDI-II, Beck Depression Inventory version-2nd edition; BAI, Beck Anxiety Inventory; GSAD, generalized social anxiety disorder subjects; HC, healthy controls; LSAS, Liebowitz Social Anxiety Scale; STAI, State-Trait Anxiety Inventory.

Note: Data are expressed as mean ( \pm SD; standard deviation); means with different subscripts (ie, a or b) across rows were significantly different in pairwise comparisons ( $p<0.05$, Tukey's honestly significant difference test for continuous variables).

Data missing from two GSAD and one HC subjects. 
history of any psychiatric disorder. All participants were non-smokers, were not taking prescription medications, and had no known allergies. During study screening, participants completed a brief medical examination. All participants provided written informed consent after explanation of the protocol, as approved by the Monash University Human Research Ethics Committee.

\section{Procedure}

The study was a within-subjects, randomized, double-blind, PBO-controlled, OXT administration study. Each participant completed two fMRI scan sessions (order randomized) where they received either intranasal spray of OXT (24 IU/ $40.32 \mu \mathrm{g}$ ) or PBO (all ingredients except for the peptide). Sessions were separated by a minimum of 7 days to allow for proper wash-out. Participants were instructed to fast for 1-h before each session, and not to ingest any caffeine or alcohol the day of the scan. The intranasal puffs were selfadministered in an alternating order with a 45-s break between puffs (total of three sprays per nostril). The fMRI scan began $45 \mathrm{~min}$ post inhalation of OXT or PBO, and lasted for $30 \mathrm{~min}$. This time frame is consistent with prior OXT administration studies and coincides with the expected timing of OXT's physiological effects (eg, Domes et al, 2007; Kirsch et al, 2005).

\section{Emotional Face Matching Task (EFMT)}

The EFMT task has previously been described in detail elsewhere (Labuschagne et al, 2010), and has been used in previous pharmaco-fMRI studies to probe drug effects on amygdala activation (Kirsch et al, 2005; Paulus et al, 2005; Phan et al, 2008; Labuschagne et al, 2010) and amygdala functional connectivity (Gorka et al, 2013). Briefly, using a block-design, participants were shown three faces and asked to select which of the two faces at the bottom of the screen matched the facial expression of the target face at the top of the screen. The target face and the congruent probe face always displayed one of three expressions (angry, fearful, or happy) and the other (ie, incongruent) probe face always displayed a neutral expression. Face matching trials were interspersed with a 'control task' where participants were asked to match simple geometric shapes (ie, circles, rectangles, or triangles) similar to the instructions above. The paradigm consisted of 18 experimental blocks, each containing four sequential matching trials of 3-s presentation (ie, 12-s blocks). There were nine blocks of matching emotional faces (three blocks of each target expression of fearful, angry, and happy), interleaved with nine blocks of matching shapes, counterbalanced across two runs. The total task time was 6-min. During the task, participants made responses by pressing the left or right response buttons with their dominant hand. These responses provided a measure of participants' response accuracy and reaction time. As noted, the planned fMRI analyses focused on matching fearful faces and shapes. Behavioral performances of the participants on the EFMT task were previously reported in Labuschagne et al (2010). There were no main effects of group or group $\times$ drug interactions on accuracy or response times when matching fearful faces or shapes (all $p s>0.05$ ).

\section{Brain Imaging}

Brain images were acquired with a $3 \mathrm{~T}$ Siemens Tim Trio scanner using a 12-channel head coil. Functional gradientecho echo-planar imaging data depicting BOLD contrast were acquired during the experimental task $(\mathrm{TE}=40 \mathrm{~ms}$, $\mathrm{TR}=3000 \mathrm{~ms}$, flip-angle $=90^{\circ}$, FoV $=210 \mathrm{~mm}, 64 \times 64 \mathrm{ma}-$ trix, 44 contiguous $3 \mathrm{~mm}$ slices parallel to the hippocampus, and interleaved). Whole brain T1-weighted anatomical reference images were also acquired from all participants for precise anatomical localization and normalization $\left(\mathrm{TE}=2.15 \mathrm{~ms}, \mathrm{TR}=1900 \mathrm{~ms}\right.$, flip-angle $=9^{\circ}, \mathrm{FoV}=256 \mathrm{~mm}$, 176 sagittal slices, $1 \mathrm{~mm}$ slice thickness, perpendicular to the AC-PC line).

\section{Functional MRI Data Analyses}

All data previously processed using Statistical Parametric Mapping software SPM2 (Wellcome Trust Centre for Neuroimaging, London, UK; Labuschagne et al, 2010) were re-processed and re-analyzed using updated SPM8 software. Images were spatially realigned to correct for head motion, slice-time corrected, warped to standardized Montreal Neurological Institute (MNI) space using the participant's mean functional image, resampled to $2 \mathrm{~mm}^{3}$ voxels, and smoothed with an $8 \mathrm{~mm}^{3}$ kernel to minimize noise and residual differences in gyral anatomy. The general linear model was applied to the time series, convolved with the canonical hemodynamic response function and with a 128 -s high-pass filter. Condition effects were modeled with box-car regressors representing the occurrence of each block type. Effects were estimated at each voxel, and for each subject. Individual contrast maps for fearful faces (> shapes) were created for each person, and each session.

We first confirmed using a whole-brain, 2 (session: $\mathrm{PBO}$, OXT) $\times 2$ (group: HC, GSAD) analysis of variance (ANOVA) that consistent with Labuschagne et al (2010), there was a significant group $\times$ drug interaction on left amygdala reactivity to fearful faces (peak MNI coordinates: $(-24,-2,-22), Z=2.77, k=81, p<0.05$, small volume corrected), such that OXT attenuated (or normalized) the hyperactive amygdala reactivity to fearful faces in GSAD patients. There was no group $\times$ drug interaction for right amygdala reactivity to fearful faces.

To examine functional coupling, we used a generalized form of context-dependent psychophysiological interaction analyses (gPPI; http://brainmap.wisc.edu/PPI, McLaren et al, 2012). The left and right amygdala were used as the seeds of interest and created using anatomically derived masks (MARINA: http://www.bion.de/Marina.htm; Walter et al, 2003), defined by atlas-based boundaries (TzourioMazoyer et al, 2002). The volumes of the masks were $1760 \mathrm{~mm}^{3}$ on the left and $1984 \mathrm{~mm}^{3}$ on the right, and the same masks were applied to all subjects' data. The de-convolved time series from the amygdala was extracted for each subject to create the physiological variable. The condition onset times for fearful faces, angry faces, happy faces, and shapes were separately convolved with the canonical hemodynamic response function for each condition, creating the psychological regressors. The interaction terms (PPIs) were computed by multiplying the time series from the psychological regressors (angry/fearful/happy) 
with the physiological variable. To examine the effect of the interaction terms, activity within the amygdala was regressed on a voxel-wise basis against the interaction, with the physiological and psychological variables serving as regressors of interest.

The individual fearful faces ( $>$ shapes) contrast images were entered into separate second-level 2 (group) $\times 2$ (drug) ANOVAs for the left and right amygdala to determine whether there were any group $\times$ drug interactions on functional connectivity. In order to conduct an exploratory whole-brain analysis, we initially set the significance at $p<0.005$ (uncorrected) with a cluster extent threshold of greater than 50 contiguous voxels to balance between type I and type II errors to generate results for subsequent hypothesis testing (Lieberman and Cunningham, 2009). Next, for a priori regions of interest, we restricted our analyses to two main areas (anterior to middle cingulate and insula) both previously shown to exhibit aberrant functional connectivity to the amygdala in GSAD (Etkin and Wager, 2007; Prater et al, 2013) and affected by OXT (Dodhia et al, 2014; Rilling et al, 2012; Sripada et al, 2013). Specifically, we applied an anatomically derived (AAL atlas) partial brain mask of the anterior and middle cingulate and bilateral insula to our data ( earch volume $=84 \mathrm{~cm}^{3}$, encompassing 10485 voxels). Cluster-based significance thresholding was used to adjust for multiple comparisons within the search volume. Based on simulations (10000 iterations) performed with 3DClustSim, an adaptation of AlphaSim (http://afni.nimh.nih.gov/pub/dist/doc/program help/3dClustSim.html), a family-wise error correction at $\alpha<0.05$ is achieved with a voxel threshold of $p<0.005$ and a cluster size of at least 60 contiguous voxels for the left amygdala model and 62 contiguous voxels for the right amygdala model. Of note, the same search mask was applied to both models, but separate thresholds were calculated given differences in estimated smoothness. We extracted parameter estimates/ $\beta$ weights (connectivity values, arbitrary units) from $5 \mathrm{~mm}$-radius spheres surrounding peak activations for each subject to conduct post hoc paired and independent samples $t$-tests and determine directionality.

\section{RESULTS}

\section{Amygdala Functional Connectivity}

gPPI functional connectivity analyses indicated a significant group $\times$ drug interaction for left amygdala to bilateral insula (right $\mathrm{MNI}(44,6,-2), Z=4.16, k=627, p<0.05$, corrected; left MNI $(-46,12,-8), Z=3.84, k=129$, $p<0.05$, corrected) and middle cingulate/dorsal anterior cingulate gyrus (mid/dACC) connectivity (MNI $(-12,0$, $42) ; Z=3.70, k=336, p<0.05$, corrected). Post hoc comparisons of extracted gPPI parameter estimates of connectivity from insula and mid/dACC are presented in Table 2 and displayed in Figure 1. In all three cases, GSAD subjects had significantly less functional connectivity than $\mathrm{HC}$ subjects on PBO. However, on OXT, this group difference in connectivity was no longer evident. In fact, on OXT, GSAD $(>\mathrm{HC}$ ) subjects displayed enhanced functional connectivity (ie, the pattern of reduced connectivity on PBO was reversed on OXT). For completeness, and to obviate bias, we report all main effects of group, main effects of drug, and group $\times$ drug interactions from the left and right amygdala gPPI ANOVAs across the entire brain (Table 3 ).

\section{Additional Analyses}

Post-hoc we conducted analyses to determine whether amygdala reactivity to fearful faces on PBO (a potential neuronal endophenotype for arousal/anxiety; Shin and Liberzon, 2010) was correlated with OXT-induced changes in amygdala functional connectivity. We found that greater left amygdala reactivity was associated with greater magnitude of OXT-induced changes in left amygdala-right insula connectivity $(r=0.46, p=0.01)$. We also conducted correlational analyses between self-reported anxiety measures and our amygdala connectivity findings, within GSAD subjects, using measures of social anxiety severity (ie, LSAS scores) and state and trait anxiety levels (ie, State-Trait Anxiety Inventory scores; Spielberger et al, 1983). Results indicated that within GSAD individuals, greater state anxiety on PBO scan day was correlated with less left amygdala-mid/dACC functional connectivity on $\mathrm{PBO}(r=-0.56, p=0.02)$. Moreover, within GSAD individuals, greater LSAS performance-based social anxiety scores were correlated with greater magnitude of OXT-induced changes in left amygdala-left insula functional connectivity $(r=0.55$, $p=0.03)$. A similar pattern of results was found with LSAS total scores, albeit at a trend level $(r=0.38, p=0.12)$. These findings are illustrated in Figure 2. Of note, as has been published elsewhere, there were no group $\times$ session, group $\times$ time (pre-, post-ingestion), or group $\times$ session $\times$ time interactions on mood (eg, anxiety) or self-reported drug effects (eg, calmness, alertness, feel high; Labuschagne et al, 2010, 2012).

\section{DISCUSSION}

The aim of the current study was to examine the effects of OXT on amygdala functional connectivity during the

Table 2 Post-hoc Between- and Within-Group Comparisons of Amygdala Functional Connectivity

\begin{tabular}{|c|c|c|c|}
\hline Functional connectivity & PBO comparison (GSAD vs HC) & OXT comparison (GSAD vs HC) & PBO vs OXT GSAD subject \\
\hline L Amygdala- L Insula & $t(3 \mathrm{I})=-2.86, p=0.0 \mathrm{I}^{\mathrm{a}}$ & $t(31)=2.56, p=0.02^{\mathrm{a}}$ & $t(15)=2.57, p=0.02^{\mathrm{a}}$ \\
\hline L Amygdala-R Insula & $t(31)=-3.10, p<0.01^{a}$ & $t(31)=2.70, p=0.01^{a}$ & $t(15)=2.14, p<0.05^{\mathrm{a}}$ \\
\hline L Amygdala-Mid/dACC & $t(3 \mathrm{I})=-2.33, p=0.03^{\mathrm{a}}$ & $t(3 \mathrm{l})=3.25, p<0.0 \mathrm{l}^{\mathrm{a}}$ & $t(\mid 5)=3.81, p<0.01^{\mathrm{a}}$ \\
\hline
\end{tabular}

Abbreviations: L, left; Mid/dACC, middle cingulate/dorsal anterior cingulate cortex; R, right.

${ }^{a} p<0.05$. 


\section{Oxytocin functional connectivity}

SM Gorka et al

Table 3 Whole-Brain Results for the gPPI Functional Connectivity ANOVA for Left and Right Amygdala at $p<0.005$ and $>50$ Contiguous Voxels

\begin{tabular}{|c|c|c|c|c|c|c|}
\hline \multirow[t]{2}{*}{ Seed } & \multirow[t]{2}{*}{ Region } & \multicolumn{3}{|c|}{ MNI coordinates } & \multirow[t]{2}{*}{ Voxels } & \multirow[t]{2}{*}{ F-Score } \\
\hline & & $x$ & $Y$ & $\mathbf{Z}$ & & \\
\hline \multirow[t]{6}{*}{ Left AMYG } & R Insula & 44 & 6 & -2 & 627 & 21.78 \\
\hline & L Superior Temporal Gyrus & -44 & -58 & 12 & 548 & 18.52 \\
\hline & R Supp Motor Area & 14 & -12 & 64 & 545 & 16.54 \\
\hline & L Rolandic Operculum & -50 & -2 & 2 & 57 & 14.34 \\
\hline & R Putamen & 18 & 6 & -6 & 68 & 13.40 \\
\hline & R Precentral Gyrus & 48 & 0 & 46 & 54 & 12.86 \\
\hline Right AMYG & R Superior Temporal Gyrus & 40 & -36 & 18 & 99 & 16.86 \\
\hline \multicolumn{7}{|c|}{ Main effect of drug } \\
\hline \multirow[t]{5}{*}{ Left AMYG } & L Medial Frontal Gyrus & -10 & 40 & 38 & 492 & 17.20 \\
\hline & R Postcentral Gyrus & 38 & -28 & 54 & 127 & 14.22 \\
\hline & R Middle Temporal Gyrus & 66 & -30 & 4 & 99 & 13.62 \\
\hline & R Heschl & 58 & -6 & 6 & 62 & 11.79 \\
\hline & R Superior Frontal Gyrus & 16 & 50 & 32 & 59 & 10.52 \\
\hline \multirow[t]{4}{*}{ Right AMYG } & R Rolandic Operculum & 48 & -26 & 22 & 786 & 20.33 \\
\hline & R Pons & 2 & -34 & -30 & $|5|$ & $|8.3|$ \\
\hline & R Middle Frontal Gyrus & 28 & -12 & 50 & 777 & 17.36 \\
\hline & L Parahippocampal Gyrus & -26 & -52 & -8 & 56 & 17.22 \\
\hline \multicolumn{7}{|c|}{ Main effect of group } \\
\hline \multirow[t]{3}{*}{ Left AMYG } & R Lingual Gyrus & 18 & -84 & -12 & 117 & 16.37 \\
\hline & L Middle Occipital Gyrus & -32 & -72 & 6 & 104 & 15.13 \\
\hline & L Fusiform Gyrus & -32 & -72 & -16 & 140 & 12.02 \\
\hline \multirow[t]{7}{*}{ Right AMYG } & L Declive & -8 & -66 & -22 & 90 & 20.88 \\
\hline & R Parietal Lobe & 24 & -44 & 32 & 113 & 19.00 \\
\hline & L Frontal Lobe & -28 & 18 & 18 & 373 & 17.89 \\
\hline & L Cuneus & -24 & -78 & 12 & 181 & 17.15 \\
\hline & Mid Cingulate Gyrus & -16 & -2 & 28 & 205 & 16.34 \\
\hline & L Posterior Cingulate Gyrus & -16 & -44 & 34 & 66 & 16.12 \\
\hline & R Cuneus & 20 & -82 & 10 & 166 & 13.49 \\
\hline
\end{tabular}

Abbreviations: AMYG, amygdala; gPPI, generalized psychophysiological interaction; L, left; MNI, Montreal Neurological Institute; R, right.

Results at $p<0.005$; uncorrected, $>50$ voxel minimum.

processing of fearful faces in GSAD subjects and HCs. Results indicated that on PBO, GSAD subjects exhibited reduced functional connectivity, relative to $\mathrm{HC}$ subjects, between amygdala-bilateral insula and amygdala-mid/ dACC. This pattern of reduced functional connectivity was no longer evident on OXT and individuals with GSAD exhibited greater functional connectivity compared with HC subjects. This suggests that OXT attenuates amygdala reactivity while simultaneously enhancing functional connectivity between the amygdala and the insula and 
a
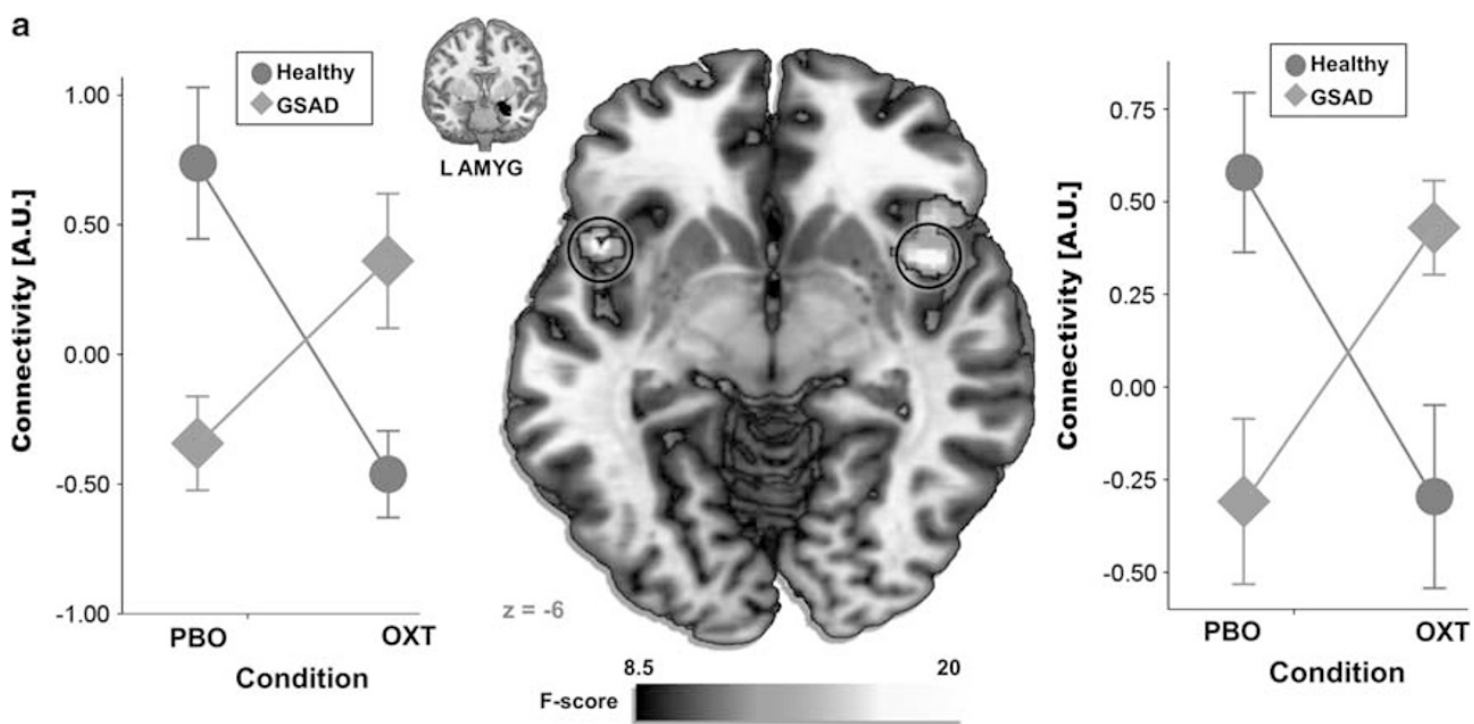

b
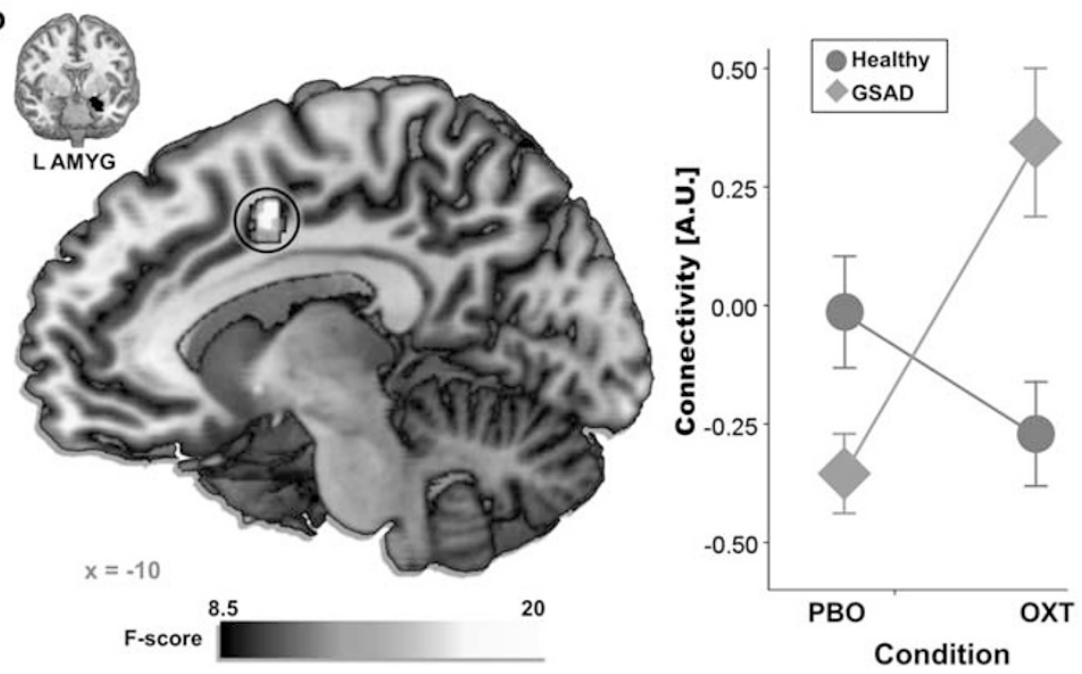

Figure I Voxel-wise statistical F-maps displayed on canonical brains illustrating significant group by drug interactions. Line graphs illustrating extracted connectivity parameter estimates during the oxytocin and placebo (PBO) conditions for GSAD and HC subjects; (a) left amygdala-left insula and left amygdala-right insula; (b) left amygdala-mid/dACC. GSAD, generalized social anxiety disorder; HC, healthy control; mid/dACC, middle cingulate/dorsal anterior cingulate cortex; OXT, oxytocin condition. A full-colour version of this figure is available at Neuropsychopharmacology journal online.

mid/dACC-regions heavily implicated in processing socioemotional stimuli.

The current pattern of results is noteworthy given that prior studies have shown reduced or disrupted amygdalainsula and amygdala-dACC connectivity to threatening faces in individuals with GSAD (Prater et al, 2013). Moreover, higher levels of social inhibition, defined as the tendency to withdrawal from people or avoid social situations, is associated with reduced connectivity between the left amygdala and the insula, dACC, and hippocampus (Blackford et al, 2014). These findings suggest that OXT may impact anxiety by 'normalizing' (ie, enhancing reduced connectivity) coupling between the amygdala, insula, and ACC. In support of these ideas, we found that greater amygdala reactivity to fearful faces (an indicator of anxious responding) was associated with less amygdala-insula functional connectivity at baseline. In addition, within GSAD subjects, greater state anxiety was related to less baseline
amygdala-mid/dACC connectivity and greater performancebased social anxiety was associated with the magnitude of OXT's effects on amygdala-insula connectivity. These findings together suggest that reduced amygdala to insula and mid/dACC functional connectivity in response to fearful stimuli may be an indicator of maladaptive or anxious responding, whereas enhanced connectivity may reflect 'normal' or typical emotional processing.

There are several potential mechanisms that may explain the current pattern of results. First, resting-state studies have suggested that the insula, dACC, and amygdala are part of a 'salience network' that identifies the most emotionally salient information among internal and external stimuli (eg, interoceptive awareness) and uses it to guide behavior (Seeley et al, 2007). Cross-talk between these regions is necessary to execute these functions and reduced connectivity may reflect dysfunctional salience processing of threat stimuli. Related to the broader social phobia 

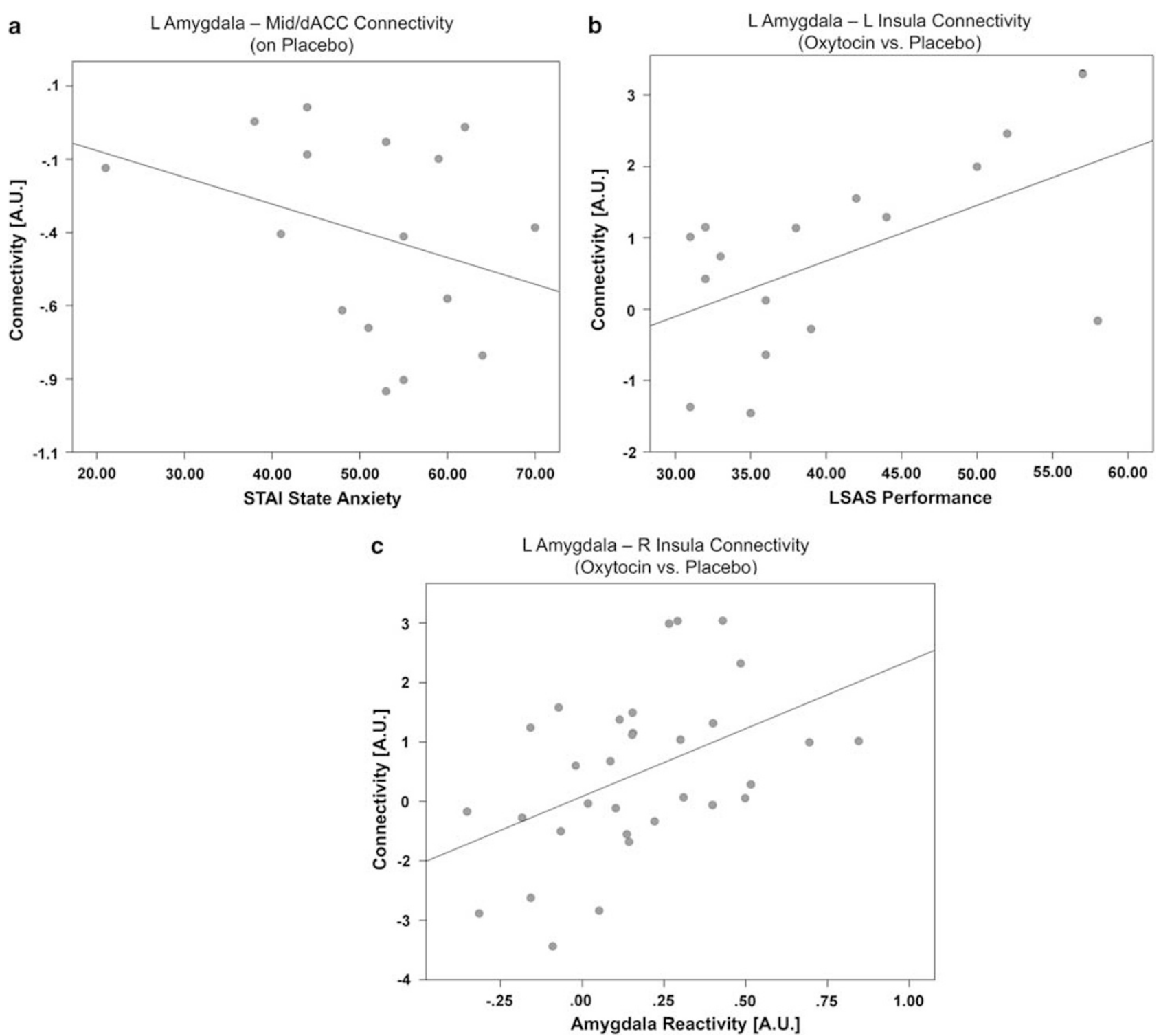

Figure 2 Scatter plot of correlation between: (a) left amygdala-mid/dACC functional connectivity on placebo and STAI state anxiety scores within GSAD subjects; (b) left amygdala-right insula functional connectivity during oxytocin (> placebo) and LSAS performance scores within GSAD subjects; (c) left amygdala-left insula functional connectivity during oxytocin (> placebo) and left amygdala reactivity on placebo within all subjects. L, left; LSAS, Liebowitz Social Anxiety Scale; Mid/dACC, middle cingulate/dorsal anterior cingulate cortex; R, right; STAI, State-Trait Anxiety Inventory.

literature, it is possible that dysfunctional salience processing underlies maladaptive attentional biases for socialevaluative stimuli that are characteristic of GSAD (Asmundson and Stein, 1994). Notably, these three regions in particular-amygdala, insula, and ACC-are increasingly implicated in the pathophysiology of GSAD (Etkin and Wager, 2007; Marazziti et al, 2014; Miskovic and Schmidt, 2012).

It is also possible that the current findings are related to the broader functions of insula and mid/dACC functional connectivity with the amygdala. The insula has direct projections to the amygdala, and functional coupling between these areas is necessary for the regulation of autonomic responses and the awareness of interoceptive states (Craig, 2009). As such, reduced connectivity may reflect diminished insula modulatory influences on amygdala reactivity, which could impair interoception and potentiate autonomic anxiety responses during social threat. Enhanced connectivity could therefore have the opposite effect and increase autonomic regulation. Of note, Rilling et al (2012) also found that OXT enhanced amygdala-insula functional connectivity and posited that this reflected an increased ability to assess internal states (eg, somatic markers) to guide decision-making. As for the mid/dACC, it is activated in response to numerous social contexts, and through functional interactions with other limbic areas, recruits regions necessary for affect regulation, directs attentional control, and ameliorates cognitive conflicts (Kerns et al, 2004). Moreover, acute OXT has been shown to reduce amygdala reactivity while enhancing connectivity to ACC and insula to infant laughter in nulliparous women and the authors suggested that increased functional connectivity 
between the amygdala and regions involved in emotion regulation may reduce negative emotional arousal while enhancing the incentive salience of the infant laughter' (Reim et al, 2012). Taken together, reduced amygdala-insula and mid/dACC functional connectivity at baseline may reflect global deficits in detecting social information and processing and modulating affective and attentional responses. It is necessary to note that although we observed these amygdala effects in the context of threat processing, interpretations of the functional relevance of OXT's actions in alleviating the symptoms or behaviors of social anxiety are speculative and future studies are needed to delineate these putative effects.

It is also important to highlight that within individuals with GSAD, OXT may not 'enhance' amygdala to insula and mid/dACC connectivity per se. Rather, the findings could be interpreted as OXT changing the direction of connectivity effects. On PBO, individuals with GSAD may exhibit strong inverse connections between the amygdala and insula and mid/dACC, whereas on OXT they exhibit strong positive connections. This would imply that hyperactive amygdala responding is coupled with reduced insula and mid/dACC activation on $\mathrm{PBO}$ and the opposite pattern on OXTreduced amygdala reactivity is coupled with enhanced insula and mid/dACC activation. Importantly, if this were indeed the case, many of the interpretations discussed above would similarly apply. Inverse connectivity may reflect aberrant interactions between these socio-emotional regions, leading to anxious responding and/or deficits in social processing. Conversely, positive connectivity may be an indicator of adaptive functional interactions which facilitate social recognition and affect modulation.

It is interesting that the aforementioned effects of OXT are specific to individuals with GSAD and differ from that observed in healthy volunteers. In fact, OXT reduces amygdala connectivity to insula and mid/dACC in HC subjects. This finding of divergent effects is consistent with the two other OXT administration studies from our group (Dodhia et al, 2014; Labuschagne et al, 2010) and underscores that acute administration of OXT can be influenced by individual differences in anxiety psychopathology. It is likely that this phenomenon has contributed to some of the mixed findings in the OXT literature (see Meyer-Lindenberg et al, 2011; but also Grillon et al, 2013). Together, these findings suggest that the relation between OXT and anxious responding is nuanced and that OXT's effects may be most consistent in the context of dysfunctional physiological states. However, future work is greatly needed to examine this question and to elucidate the moderators and mediators of OXT's acute effects on typical and atypical brain function.

Although these results extend the literature on the neural effects of OXT, there are a few limitations worth noting. First, although a within-subjects study, the sample size was small and this likely reduced statistical power. Second, our sample was restricted to males, given the role of sex hormones on OXT neural effect's (Domes et al, 2010), and may not generalize to females. Third, gPPI analyses are correlational and therefore directionality between amygdala and the insula and mid/dACC cannot be inferred. Likewise, functional relevance of OXT's effects on amygdala reactivity and functional connectivity remain unclear and more work is needed to link these effects to functional outcomes. Future studies are also needed to determine whether the current findings are specific to fear or similarly apply to other social stimuli (eg, anger, disgust), and how these findings relate to OXT induced changes in GABAergic transmission within the amygdala.

In sum, the results suggest that within individuals with GSAD, OXT simultaneously dampens amygdala reactivity and enhances amygdala functional connectivity with the insula and mid/dACC during the processing of fearful faces. These neural effects may have broad pro-social implications such as enhancing the integration and modulation of social responses. These data add to the growing evidence of OXT's acute effects on reactivity and connectivity within a socioemotional, salience brain network in GSAD, and prompt further studies to further investigate specific mechanisms of OXT's actions in anxious states and social behaviors.

\section{FUNDING AND DISCLOSURE}

The authors declare no conflict of interest.

\section{ACKNOWLEDGEMENTS}

This research was funded by an Independent Investigator Award to PJN from the Brain and Behavior Research Foundation (formally National Alliance for Research in Schizophrenia and Depression (NARSAD)). PJN was an employee of GSK and UCB Pharma during the conduct of the study but received no funding from either company for the study. AGW was supported by an Australian Rotary Health fellowship (Geoffrey Betts Award) but ARH had no role in the current study.

\section{REFERENCES}

American Psychiatric Association (1994). Diagnostic and Statistical Manual of Mental Disorders, 4th edn. American Psychiatric Association: Washington, DC.

Asmundson GJ, Stein MB (1994). Selective processing of social threat in patients with generalized social phobia: evaluation using a dot-probe paradigm. J Anxiety Disord 8: 107-117.

Baumgartner T, Heinrichs M, Vonlanthen A, Fischbacher U, Fehr E (2008). Oxytocin shapes the neural circuitry of trust and trust adaptation in humans. Neuron 58: 639-650.

Bethlehem RA, van Honk J, Auyeung B, Baron-Cohen S (2013). Oxytocin, brain physiology, and functional connectivity: a review of intranasal oxytocin fMRI studies. Psychoneuroendocrinology 38: 962-974.

Blackford JU, Clauss JA, Avery SN, Cowan RL, Benningfield MM, VanDerKlok RM (2014). Amygdala-cingulate intrinsic connectivity is associated with degree of social inhibition. Biol Psychol 99: $15-25$.

Craig AD (2009). How do you feel-now? The anterior insula and human awareness. Nat Rev Neurosci 10: 59-70.

Ditzen B, Schaer M, Gabriel B, Bodenmann G, Ehlert U, Heinrichs M (2009). Intranasal oxytocin increases positive communication and reduces cortisol levels during couple conflict. Biol Psychiatry 65: $728-731$

Dodhia S, Hosanagar A, Fitzgerald DA, Labuschagne I, Wood AG, Nathan PJ et al (2014). Modulation of resting-state amygdalafrontal functional connectivity by oxytocin in generalized social anxiety disorder. Neuropsychopharmacology 39: 2061-2069. 
Domes G, Heinrichs M, Gläscher J, Büchel C, Braus DF, Herpertz SC (2007). Oxytocin attenuates amygdala responses to emotional faces regardless of valence. Biol Psychiatry 62: 1187-1190.

Domes G, Lischke A, Berger C, Grossmann A, Hauenstein K, Heinrichs $\mathrm{M}$ et al (2010). Effects of intranasal oxytocin on emotional face processing in women. Psychoneuroendocrinology 35: 83-93.

Etkin A, Wager T (2007). Functional neuroimaging of anxiety: a meta-analysis of emotional processing in PTSD, social anxiety disorder, and specific phobia. Am J Psychiatry 164: 1476-1488.

Foa EB, Gilboa-Schechtman E, Amir N, Freshman M (2000). Memory bias in generalized social phobia: remembering negative emotional expressions. J Anxiety Disord 14: 501-519.

Gorka SM, Fitzgerald DA, King AC, Phan KL (2013). Alcohol attenuates amygdala-frontal connectivity during processing social signals in heavy social drinkers. Psychopharmacology 229: $141-154$.

Grillon C, Krimsky M, Charney DR, Vytal K, Ernst M, Cornwell B (2013). Oxytocin increases anxiety to unpredictable threat. Mol Psychiatry 18: 958-960.

Harmer CJ, Mackay CE, Reid CB, Cowen PJ, Goodwin GM (2006). Antidepressant drug treatment modifies the neural processing of nonconscious threat cues. Biol Psychiatry 59: 816-820.

Hermann C, Ziegler S, Birbaumer N, Flor H (2002). Psychophysiological and subjective indicators of aversive Pavlovian conditioning in generalized social phobia. Biol Psychiatry 52: 328-337.

Huber D, Veinante P, Stoop R (2005). Vasopressin and oxytocin excite distinct neuronal populations in the central amygdala. Science 308: 245-248.

Kerns JG, Cohen JD, MacDonald AW, Cho RY, Stenger VA., Carter CS (2004). Anterior cingulated conflict monitoring and adjustments in control. Science 303: 1023-1026.

Kirsch P, Esslinger C, Chen Q, Mier D, Lis S, Siddhanti S et al (2005). Oxytocin modulates neural circuitry for social cognition and fear in humans. J Neurosci 25: 11489-11493.

Kosfeld M, Heinrichs M, Zak PJ, Fischbacher U, Fehr E (2005). Oxytocin increases trust in humans. Nature 435: 673-676.

Labuschagne I, Phan KL, Wood A, Angstadt M, Chua P, Heinrichs $M$ et al (2010). Oxytocin attenuates amygdala reactivity to fear in generalized social anxiety disorder. Neuropsychopharmacology 35: 2403-2413.

Labuschagne I, Phan KL, Wood A, Angstadt M, Chua P, Heinrichs $M$ et al (2012). Medial frontal hyperactivity to sad faces in generalized social anxiety disorder and modulation by oxytocin. Int J Neuropsychopharmacol 15: 883-896.

Lieberman MD, Cunningham WA (2009). Type I and Type II error concerns in fMRI research: Rebalancing the scale. Soc Cogn Affect Neurosci 4: 423-428.

Liebowitz MR (1987). Social phobia. Mod Probl Pharmacopsychiatry 22: 141-173.

Marazziti D, Abelli M, Baroni S, Carpita B, Ramacciotti CE, Dell'Osso L (2014). Neurobiological correlates of social anxiety disorder: an update. CNS Spectrums (e-pub ahead of print).

McLaren DG, Ries ML, Xu G, Johnson SC (2012). A generalized form of context-dependent psychophysiological interactions (gPPI): a comparison to standard approaches. Neuroimage 61: $1277-1286$.

Meyer-Lindenberg A, Domes G, Kirsch P, Heinrichs M (2011). Oxytocin and vasopressin in the human brain: Social neuropeptides for translational medicine. Nat Rev Neurosci 12: 524-538.

Miskovic V, Schmidt LA (2012). Social fearfulness in the human brain. Neurosci Biobehav R 36: 459-478.

Mogg K, Philippot P, Bradley BP (2004). Selective attention to angry faces in clinical social phobia. J Abnorm Psychol 113: 160.
Paulus MP, Feinstein JS, Castillo G, Simmons AN, Stein MB (2005). Dose-dependent decrease of activation in bilateral amygdala and insula by lorazepam during emotion processing. Arch Gen Psychiatry 62: 282-288.

Phan KL, Angstadt M, Golden J, Onyewuenui I, Povpovska A, de Wit H (2008). Cannabinoid modulation of amygdala reactivity to social signals of threat in humans. J Neurosci 28: 2313-2319.

Phan KL, Fitzgerald DA, Nathan PJ, Tancer ME (2006). Association between amygdala hyperactivity to harsh faces and severity of social anxiety in generalised social phobia. Biol Psychiatry 59: 424-429.

Prater KE, Hosanagar A, Klumpp H, Angstadt M, Phan KL (2013). Aberrant amygdala-frontal cortex connectivity during perception of fearful faces and at rest in generalized social anxiety disorder. Depress Anxiety 30: 234-241.

Riem MM, van IJzendoorn MH, Tops M, Boksem MA, Rombouts SA, Bakermans-Kranenburg MJ (2012). No laughing matter: intranasal oxytocin administration changes functional brain connectivity during exposure to infant laughter. Neuropsychopharmacology 37: 1257-1266.

Rilling JK, DeMarco AC, Hackett PD, Thompson R, Ditzen B, Patel $\mathrm{R}$ et al (2012). Effects of intranasal oxytocin and vasopressin on cooperative behavior and associated brain activity in men. Psychoneuroendocrinology 37: 447-461.

Roelofs K, van Peer J, Berretty E, de Jong P, Spinhoven P, Elzinga BM (2009). HPA-axis hyperresponsiveness is associated with increased social avoidance behavior in social phobia. Biol Psychiatry 65: 336-343.

Schultz LT, Heimberg RG (2008). Attentional focus in social anxiety disorder: potential for interactive processes. Clin Psychol Rev 28: 1206-1221.

Seeley WW, Menon V, Schatzberg AF, Keller J, Glover GH, Kenna $\mathrm{H}$ et al (2007). Dissociable intrinsic connectivity networks for salience processing and executive control. J Neurosci 27: 2349-2356.

Shin LM, Liberzon I (2010). The neurocircuitry of fear, stress, and anxiety disorders. Neuropsychopharmacology 35: 169-191.

Spielberger CD, Gorsuch RL, Lushene RE (1983). Manual for the State-Trait Anxiety Inventory. Consulting Psychologist Press: Palo Alto, CA.

Sripada CS, Phan KL, Labuschagne I, Welsh R, Nathan PJ, Wood AG (2013). Oxytocin enhances resting-state connectivity between amygdala and medial frontal cortex. Int J Neuropsychopharmacology 16: 255-260.

Taylor S, Abramowitz JS, McKay D (2012). Non-adherence and non-response in the treatment of anxiety disorders. J Anxiety Disord 26: 583-589.

Tzourio-Mazoyer N, Landeau B, Papathanassiou D, Crivello F, Etard O, Delcroix N et al (2002). Automated anatomical labeling of activations in SPM using a macroscopic anatomical parcellation of the MNI MRI single-subject brain. Neuroimage 15: 273-289.

Van Den Heuvel MP, Hulshoff Pol HE (2010). Exploring the brain network: a review on resting-state fMRI functional connectivity. Eur Neuropsychopharmacology 20: 519-534.

Walter B, Blecker C, Kirsch P, Sammer G, Schienle A, Stark R et al (2003). Marina: An easy to use tool for the creation of masks for interest analyses. Neuroimage 19: S47.

WHO (1997). World Health Organization Composite International Diagnostic Interview (CIDI, Version 2.1), World Health Organization: Geneva.

Windle RJ, Shanks N, Lightman SL, Ingram CD (1997). Central oxytocin administration reduces stress-induced corticosterone release and anxiety behavior in rats 1. Endocrinology 138: 2829-2834. 